Portland State University

PDXScholar

\title{
Brief Report: Medication Sharing Is Rare Among African HIV-1 Serodiscordant Couples Enrolled in an Efficacy Trial of Oral Pre-exposure Prophylaxis (PrEP) for HIV-1 Prevention
}

\author{
Kerry A. Thomson \\ University of Washington \\ Jessica Haberer \\ Massachusetts General Hospital \\ Mark A. Marzinke \\ Johns Hopkins University \\ Andrew Mujugira \\ Makerere University \\ Craig Hendrix Follow this and additional works at: https://pdxscholar.library.pdx.edu/sph_facpub \\ Johns Hopkins University \\ Part of the Community Health and Preventive Medicine Commons \\ Lee us moxt page for additional authors
}

\section{Citation Details}

Thomson, K. A., Haberer, J. E., Marzinke, M. A., Mujugira, A., Hendrix, C. W., Celum, C., \& ... Baeten, J. M. (2017). Brief Report: Medication Sharing Is Rare Among African HIV-1 Serodiscordant Couples Enrolled in an Efficacy Trial of Oral Pre-exposure Prophylaxis (PrEP) for HIV-1 Prevention. Journal Of Acquired Immune Deficiency Syndromes (1999), 75(2), 184-189.

This Article is brought to you for free and open access. It has been accepted for inclusion in OHSU-PSU School of Public Health Faculty Publications and Presentations by an authorized administrator of PDXScholar. Please contact us if we can make this document more accessible: pdxscholar@pdx.edu. 


\section{Authors}

Kerry A. Thomson, Jessica Haberer, Mark A. Marzinke, Andrew Mujugira, Craig Hendrix, Connie Celum,

Patrick Ndase, Alan Ronald, David Bangsberg, and Jared Baeten 


\title{
Medication Sharing Is Rare Among African HIV-1 Serodiscordant Couples Enrolled in an Efficacy Trial of Oral Pre-exposure Prophylaxis (PrEP) for HIV-1 Prevention
}

\author{
Kerry A. Thomson, MPH, * Jessica E. Haberer, MD, MS, $†$ Mark A. Marzinke, PhD, $\neq$ \\ Andrew Mujugira, MBChB, PhD, MSc, $\$$ Craig W. Hendrix, MD,\| Connie Celum, MD, MPH, $\mid$ \\ Patrick Ndase, MBChB, MPH,\# Allan Ronald, MD,** David R. Bangsberg, MD, MPH, †† and \\ Jared M. Baeten, MD, PhD, $+\neq$ for the Partners PrEP Study Team
}

\begin{abstract}
Sharing of pre-exposure prophylaxis (PrEP) medications is a concern for PrEP implementation. For HIV-1 serodiscordant couples, sharing may undermine the HIV-1 prevention benefit and also cause antiretroviral resistance if taken by HIV-1 infected partners. Within a PrEP efficacy trial among HIV-1 serodiscordant couples, we assessed the occurrence of PrEP sharing by self-report and plasma tenofovir concentrations in HIV-1 infected partners. PrEP sharing was self-reported at $<0.01 \%$ of visits, and $0 \%-1.6 \%$ of randomly selected and $0 \%$ of purposively selected specimens from HIV-1 infected participants had detectable tenofovir concentrations (median: $66.5 \mathrm{ng} / \mathrm{mL}$, range: $1.3-292 \mathrm{ng} / \mathrm{mL}$ ). PrEP sharing within HIV-1 serodiscordant couples was extremely rare.
\end{abstract}

Key Words: HIV-1, pre-exposure prophylaxis, HIV-1 serodiscordant couples, adherence, prescription drug diversion

(J Acquir Immune Defic Syndr 2017;75:184-189)

Received for publication October 12, 2016; accepted February 22, 2017. From the *Department of Epidemiology, University of Washington, Seattle, WA; †Center for Global Health and Department of Medicine, Massachusetts General Hospital, Boston, MA; $\ddagger$ Departments of Pathology and Medicine, Johns Hopkins University, Baltimore, MD; §Department of Epidemiology and Biostatistics, Makerere University, Kampala, Uganda; |Division of Clinical Pharmacology and Department of Medicine, Johns Hopkins University School of Medicine, Baltimore, MD; $\llbracket$ Departments of Global Health, Medicine, and Epidemiology, University of Washington, Seattle, WA; \#Department of Global Health, University of Washington, Seattle, WA; **Department of Medicine, University of Manitoba, Winnipeg, Canada; $\dagger$ Oregon Health and Sciences University and Portland State University School of Public Health, Portland, OR; and $+\underset{\dagger}{ }$ Departments of Global Health, Medicine, and Epidemiology, University of Washington, Seattle, WA.

Supported by the Bill and Melinda Gates Foundation (research grants OPP47674 and OOP52516) and the National Institutes of Health (research grants R01 MH095507 and P30 AI 027757).

Presented at the Conference on Retroviruses and Opportunistic Infections (CROI); February 25, 2015; Seattle, WA (abstract \#988).

The authors have no conflicts of interest to disclose.

Correspondence to: Kerry A. Thomson, MPH, Department of Epidemiology, University of Washington, 325 Ninth Avenue, Box 359927, Seattle, WA 98104 (e-mail: thomsonk@uw.edu).

Copyright (C) 2017 The Author(s). Published by Wolters Kluwer Health, Inc. This is an open-access article distributed under the terms of the Creative Commons Attribution-Non Commercial License 4.0 (CCBY-NC), where it is permissible to download, share, remix, transform, and build up the work provided it is properly cited. The work cannot be used commercially without permission from the journal.

\section{INTRODUCTION}

Pre-exposure prophylaxis (PrEP), in which an HIV-1 uninfected individual takes oral antiretrovirals to prevent HIV-1 acquisition, is recommended by the World Health Organization for populations at high risk of HIV-1, including HIV-1 serodiscorant couples. ${ }^{1}$ Open-label studies of PrEP are ongoing, and have shown high adherence and reductions in HIV-1 acquisition of $90 \%$ or more. $^{2-6}$

"Drug diversion," where HIV-1 uninfected individuals share or sell their prescribed PrEP medications, is a potential challenge for PrEP implementation. ${ }^{7-9}$ To sufficiently disrupt HIV-1 replication, antiretroviral therapy (ART) for HIV infected adults should include at least 3 drugs from 2 classes of HIV antiretrovirals. ${ }^{10}$ Use of mono or dual agent antiretrovirals prescribed as PrEP by HIV-1 infected persons could result in antiretroviral resistance due to suboptimal viral suppression and undermine the level of protection for the intended HIV-1 uninfected user due to insufficient drug concentrations. ${ }^{11}$ PrEP sharing may occur more frequently by HIV-1 uninfected persons with a known HIV-1 infected partner, particularly if their partner is not yet eligible for or experiences barriers accessing ART for their own treatment. We assessed the extent to which PrEP sharing occurred within a large cohort of HIV-1 serodiscordant couples enrolled in an efficacy trial in Africa.

\section{METHODS}

\section{Study Population}

The Partners PrEP Study was a phase III, randomized, double-blind, placebo-controlled, 3-arm clinical trial of daily oral PrEP (ClinicalTrials.gov NCT00557245). Beginning in 2008, the HIV-1 uninfected partner in 4747 HIV-1 serodiscordant couples was randomized to receive once-daily tenofovir (TDF), combination tenofovir-emtricitabine (TDF/ FTC), or matching placebo and followed for a maximum of 48 months for safety and HIV-1 seroconversion. The Partners PrEP Study was conducted at 9 clinical research sites in Kenya and Uganda; study details have been described previously. ${ }^{12-14}$ In July 2011, the Data and Safety Monitoring Board recommended discontinuation of the placebo arm due to the demonstrated $67 \%$ efficacy for HIV-1 prevention with 
TDF and $75 \%$ efficacy with TDF/FTC. ${ }^{13}$ Participants were offered the option to continue in the study and receive openlabel PrEP, with participants in the placebo arm re-randomized to TDF or TDF/FTC.

\section{Study Procedures}

This analysis includes data from the duration of study follow-up (2008-2013). HIV-1 uninfected participants attended monthly study visits. At each visit, participants were counselled on the importance of adherence to study drug and the potential danger of HIV-1 infected partners taking study drug. ${ }^{13,15}$ Plasma from HIV-1 uninfected partners was collected at enrollment, months 1,3 , and quarterly thereafter, and study exit. At enrollment, all HIV-1 infected partners were ineligible for ART based on national guidelines and selfreported not taking ART. HIV-1 infected participants attended quarterly study visits and were referred to initiate ART once they met national eligibility criteria. Plasma from HIV-1 infected participants was collected at enrollment, at 6 months intervals thereafter, and study exit. Plasma was also collected at any visit where an HIV-1 uninfected participant first tested positive for HIV, and as soon as possible from their study partner. Self-reported data on sharing study drug, including the number of tablets shared, were obtained from HIV-1 uninfected partners at monthly study visits, rerandomization, study exit, and annually from HIV-1 infected partners.

\section{Assessment of PrEP Medication Sharing}

For the current analysis, we assessed PrEP medication sharing using self-report and objective assessment through plasma tenofovir testing in 4 different groups that were selected based on potential or previously documented nonadherence to study drug (Fig. 1). Tenofovir concentrations in plasma were measured using previously described validated liquid chromatography-mass spectrometry/mass spectrometry methods. ${ }^{16,17}$ Detectable tenofovir for all analyses was defined as $\geq 0.31 \mathrm{ng} / \mathrm{mL}$; this threshold was the lower limit of quantification and has $92 \%$ specificity for at least one dose in the past week. ${ }^{17}$ Results from 2 prior analyses ${ }^{13,18}$ informed the sampling scheme for the current study, and additional testing of plasma from HIV-1 infected subjects was performed. The Ancillary Adherence Study (AAS) assessed use of study drug during the blinded phase of the trial at 3 sites in Uganda. ${ }^{15}$ In this substudy, plasma was collected from both HIV-1 uninfected and HIV-1 infected members of 1147 couples at unannounced home visits during follow-up. Group 1 was a random sample of plasma specimens collected at AAS unannounced home visits from HIV-1 infected participants $(\mathrm{n}=100)$, with the rationale that participants sharing medication may modify this behavior before clinic visits and thus an unannounced visit would be the best opportunity to identify sharing. Group 2 included specimens belonging to HIV-1 infected participants enrolled in the AAS whose HIV-1 uninfected study partner's specimen was included in a previous analysis testing 268 randomly selected home specimens and did not have detectable tenofovir concentrations $(\mathrm{n}=$ 29). ${ }^{18}$ Group 3 was derived from a case-cohort study of plasma tenofovir concentrations nested within the Partners PrEP Study, including 298 randomly-selected HIV-1 uninfected participants from the active arms, which assessed the association between PrEP use and HIV-1 protection. ${ }^{13}$ Group 3 included specimens belonging to HIV-1 infected participants whose HIV-1 uninfected study partner's specimen did not have detectable tenofovir concentrations among the 1802 randomly selected specimens in the cohort analysis $(\mathrm{n}=406) .{ }^{12,13}$ The rationale for both Groups 2 and 3 was that the absence of tenofovir in the HIV-1 uninfected partner may have been because PrEP medication was shared with the HIV-1 infected partner. Group 4 included HIV-1 infected participants whose study partner acquired HIV-1 during study follow-up $(\mathrm{n}=52)$, with the rationale that these participants were not taking sufficient PrEP to achieve
Self-reported Medication Sharing: All Participants

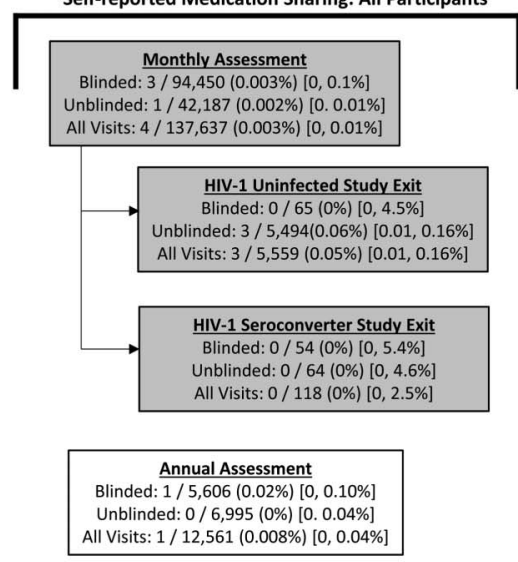

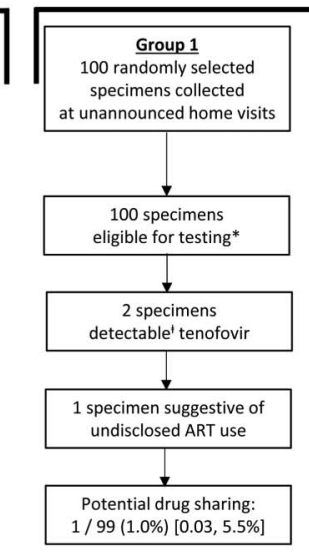

Plasma Tenofovir: Selected Groups
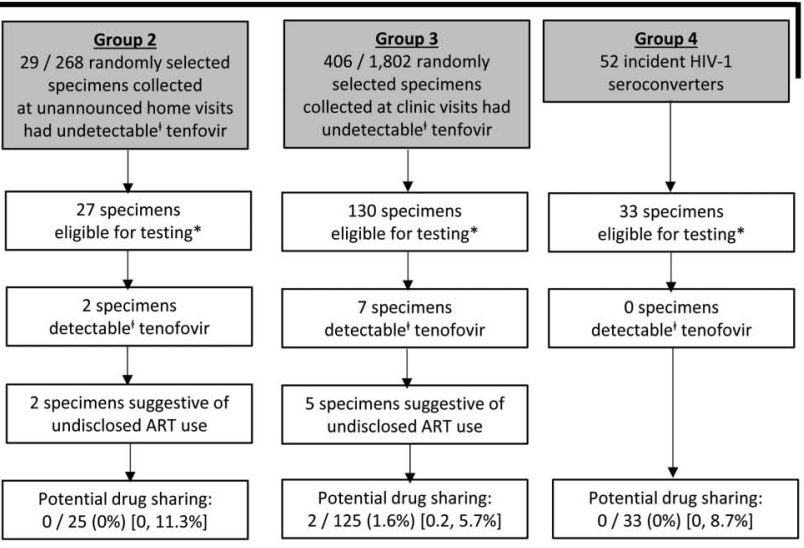

"Specimens were eligible for tenofovir testing if HIV-1 infected part Detectable tenofovir concentrations defined as $>0.31 \mathrm{ng} / \mathrm{mL}$

HIV-1 Uninfected Partner HIV-1 Infected Partner

FIGURE 1. Assessment of potential sharing of PrEP medication within HIV-1 serodiscordant couples, frequency, (\%), [95\% CI]. 
the HIV-1 prevention benefit and may have diverted PrEP to their partner.

The underlying sampling scheme for Groups 1, 2, and 3 was a random selection across the study population; within Groups 2 and 3, and for Group 4, purposeful testing was done to investigate drug sharing because uninfected partners had no tenofovir concentrations in plasma and/or acquired HIV-1 during follow-up, despite having access to active study drug. Specimens from couples who had been randomized to the trial's active arms and time periods when PrEP was dispensed (ie, excluding study drug holds or missed visits) and ART was not reported by the HIV-1 infected partner were eligible for testing. We tested the specimen from the HIV-1 infected participant that was closest to the date of undetectable tenofovir concentrations (or HIV-1 seroconversion) in their HIV-uninfected partner. For any HIV-1 infected participant with detectable tenofovir concentrations we also tested the following: an additional specimen from the same visit to rule out inadvertent specimen mix-up, plasma archived at enrollment/randomization (to assess undisclosed ART use), plasma HIV-1 RNA viral load (undetectable defined as $<80$ copies $/ \mathrm{mL}$ ), and plasma for emtricitabine.

\section{Statistical Methods}

We used SAS 9.4 (SAS Institute, Cary, NC) for descriptive analysis; $95 \%$ confidence intervals were generated with Stata 13.1 (StataCorp, College Station, TX), and onesided exact $95 \%$ confidence intervals were generated for proportions in which the numerator was zero.

\section{Ethics}

The protocols for the parent study and AAS sub-study were approved by ethics review committees at the University of Washington and each study site. All participants provided written informed consent.

\section{RESULTS}

\section{Self-Report of Medication Sharing}

Eight instances of HIV-1 infected partners using study medication were self-reported from 7 couples across 155,875 study visits $(8 / 155,875,<0.01 \%)$. Five of these instances were reported during follow-up and 3 were reported at study exit, including one partner who reported medication sharing once during follow-up and again at study exit (Fig. 1). No seroconverter reported that their study partner had used their study medication. All self-reported instances of HIV-1 infected participants taking study medication were described as a small number of pills (maximum 4) during a single study month, and there was no increase in reported drug sharing once the placebo arm was discontinued.

\section{Plasma Drug Concentrations}

Of 290 specimens tested for this analysis, a total of 11 specimens from HIV-1 infected participants had detectable tenofovir concentrations, though only 3 specimens are potential instances of drug sharing (Fig. 1). These 11 specimens came from 9 participants, none of whom self-reported drug sharing, and all were investigated further for potential drug sharing and undisclosed ART use (Table 1). Two of the 100 specimens in Group 1 had detectable tenofovir concentrations. Follow-up testing determined that Case 1 had a tenofovir concentration of $71.5 \mathrm{ng} / \mathrm{mL}$ and an undetectable HIV-1 viral load at enrollment, suggesting unreported tenofovir-containing ART use before randomization and not drug sharing thereafter. Case 2 had undetectable viral load and a very low tenofovir concentrations at month 11 (1.3 ng/ $\mathrm{mL}$ ), but no tenofovir present at enrollment. Thus, PrEP sharing may have occurred in this case and overall, potential drug sharing in Group 1 was $1.0 \%$. In Group 2, 2 specimens from the same participant had detectable tenofovir concentrations (Case 3). This couple was randomized to the TDF arm; however, the HIV-1 infected partner had both tenofovir and emtricitabine detected in a specimen collected at enrollment. Case 3 is suggestive of undisclosed ART use before study entry and unlikely PrEP sharing thereafter; therefore potential drug sharing in Group 2 was $0 \%$. In Group 3, 7 specimens from 6 participants had detectable tenofovir concentrations (Cases 4-9). Three participants randomized to the TDF/FTC arm had detectable tenofovir without emtricitabine (Case 4-6) and Case 7 had detectable tenofovir before randomization, all circumstances suggesting undisclosed ART use. Case 8 had detectable tenofovir concentrations at month 24 of follow-up and drug concentrations persisted until study exit at month 36, and Case 9 had detectable tenofovir concentrations and undetectable HIV-1 viral load at month 33. In the absence of detectable tenofovir concentrations at randomization for these 2 cases, potential drug sharing in Group 3 was $1.6 \%$. No specimens in Group 4 had detectable tenofovir and therefore potential drug sharing in this group was $0 \%$.

\section{DISCUSSION}

We found strong evidence that PrEP medication sharing was extremely infrequent among East African HIV-1 serodiscordant couples enrolled in a PrEP efficacy trial. No extended drug sharing was self-reported, and random testing and purposive sampling among groups most likely to share study drug identified only 3 couples with evidence of potential sharing of PrEP. Only one prior study has explored drug sharing within the context of a PrEP efficacy trial and also found that it was rare. Among a subset of HIV-1 uninfected women enrolled in the FEM-PrEP study, 10/224 (4\%) of women reported that they gave their study pills to someone else and described instances of selling or sharing study medication to HIV-1 infected and uninfected individuals. ${ }^{20}$ While outside the scope of the current analysis, sharing PrEP with other HIV-1 uninfected individuals who are not receiving adherence counselling and frequent HIV-1 testing is also a concern for PrEP delivery and should be explored in future studies.

For 6 HIV-1 infected partners, we found detectable drug at a time point prior to their study partner receiving PrEP and/or concentrations that were not consistent with the arm to 
Table 1. Characteristics and Plasma Drug Concentrations of HIV-1 Infected Participants With Detectable Tenofovir

\begin{tabular}{|c|c|c|c|c|c|c|c|}
\hline Case & $\begin{array}{l}\text { Testing } \\
\text { Group }\end{array}$ & Gender & $\begin{array}{c}\text { Total } \\
\text { Study } \\
\text { Months }\end{array}$ & Study Arm & $\begin{array}{l}\text { Drug Levels by Study } \\
\text { Month (ng/mL)* }\end{array}$ & $\begin{array}{l}\text { HIV-1 Viral Load } \\
\quad \text { (copies } / \mathrm{mL})\end{array}$ & $\begin{array}{l}\text { Conclusion on Drug } \\
\text { Sharing within Study } \\
\text { Partnership }\end{array}$ \\
\hline 1 & 1 & Male & 36 & TDF/FTC & $\begin{array}{l}\text { Month 0: FTC ND, TFV } 71.5 . \\
\text { Month 12: FTC NT, TFV } \\
\text { 79.2. Month 15: FTC NT, } \\
\text { TFV 72.7. }\end{array}$ & Month 0: UD. Month 12: UD. & $\begin{array}{l}\text { Detectable TFV only and no } \\
\text { FTC at randomization, } \\
\text { indicates drug could not } \\
\text { come from study partner. } \\
\text { Undisclosed ART at } \\
\text { enrollment }\end{array}$ \\
\hline 3 & 2 & Female & 36 & TDF & $\begin{array}{l}\text { Month 0: FTC 73.1, TFV } \\
\text { 27.4. Month 6: FTC ND, } \\
\text { TFV 56.1. Month 18: FTC } \\
\text { ND, TFV 41.3. }\end{array}$ & Month 0: UD. Month 12: UD. & $\begin{array}{l}\text { Detectable TFV and FTC, } \\
\text { starting at randomization, } \\
\text { indicates drug could not } \\
\text { come from study partner. } \\
\text { Undisclosed ART at } \\
\text { enrollment }\end{array}$ \\
\hline 5 & 3 & Female & 36 & $\begin{array}{l}\text { Placebo } \\
\text { TDF/FTC }\end{array}$ & $\begin{array}{l}\text { Month 0: FTC ND, TFV ND. } \\
\text { Month 24: FTC ND, TFV } \\
46.6 \text { (Rerandomization). }\end{array}$ & $\begin{array}{l}\text { Month 0: UD. Month 12: UD. } \\
\text { Month 24: UD. }\end{array}$ & $\begin{array}{l}\text { Detectable TFV only and no } \\
\text { FTC, starting at re- } \\
\text { randomization, indicates } \\
\text { drug could not come from } \\
\text { study partner. Undisclosed } \\
\text { ART use during follow-up }\end{array}$ \\
\hline 6 & 3 & Female & 33 & TDF/FTC & $\begin{array}{l}\text { Month 0: FTC ND; TFV ND. } \\
\text { Month 33: FTC ND, TFV } \\
\text { 58.1. }\end{array}$ & $\begin{array}{l}\text { Month 0: 682. Month 12: } 212 \text {. } \\
\text { Month 24: } 436 .\end{array}$ & $\begin{array}{l}\text { Detectable TFV only and no } \\
\text { FTC indicates drug could } \\
\text { not come from study } \\
\text { partner. Undisclosed ART } \\
\text { use during follow-up }\end{array}$ \\
\hline 9 & 3 & Female & 33 & TDF & $\begin{array}{l}\text { Month 0: FTC ND, TFV ND. } \\
\text { Month 27: FTC ND, TFV } \\
\text { ND. Month 30: FTC ND, } \\
\text { TFV ND. Month 33: FTC } \\
\text { ND, TFV 74.3. }\end{array}$ & $\begin{array}{l}\text { Month 0: 41,350; Month 33: } \\
\text { UD }\end{array}$ & $\begin{array}{l}\text { Potential PrEP sharing. } \\
\text { Alternative explanation } \\
\text { may be ART initiation } \\
\text { without reporting to the } \\
\text { study team }\end{array}$ \\
\hline
\end{tabular}

*Plasma tenofovir $>0.31 \mathrm{ng} / \mathrm{mL}$ is consistent with dosing within the last week, $>10 \mathrm{ng} / \mathrm{mL}$ is consistent with dosing in the previous $2-3 \mathrm{days}$, and $>40 \mathrm{ng} / \mathrm{mL}$ is the lower bound of the $95 \%$ confidence interval for directly observed dosing at steady state. ${ }^{19}$

$\dagger$ indicates plasma collected at an unannounced home visit.

ART, antiretroviral therapy; FTC, emtricitabine; TDF or TNF, tenofovir; ND, drug levels not detected $(<0.31 \mathrm{ng} / \mathrm{mL})$; NT, not tested; UD, HIV-1 RNA viral load undetectable $(<$ 80 copies $/ \mathrm{mL}$ ).

which the study couple had been randomized. Although it was not feasible to test these specimens for all possible antiretrovirals, the timing detectable tenofovir or emtricitabine strongly suggests undisclosed ART use. This finding is consistent with prior studies that have described undisclosed ART use occurring among $2.8 \%-5 \%$ of HIV-1 infected partners participating in clinical trials of HIV-1 serodiscordant couples. ${ }^{21,22}$ Undisclosed ART use could also explain the 
3 cases of potential PrEP sharing we observed in the current study.

Plasma was not always available at the exact same time point for both members of the partnership because HIV-1 uninfected participants were seen monthly and HIV1 infected partners were seen quarterly. It is possible that instances of time-limited drug sharing could have been undetected. Our study population of mutually disclosed HIV-1 serodiscordant couples enrolled in a clinical trial places some limitations on the generalizability of our findings. All participants in the Partners PrEP Study received frequent adherence counselling, including the potential for ART drug resistance if the HIV-1 infected study partner took study medication, counselling which is more intensive than in an implementation setting. Nevertheless, medication sharing was feasible in our study population.

HIV-1 serodiscordant couples have been identified as a priority for PrEP implementation. Using prospectively collected data, including self-report and objective testing of drug levels in 4 groups that represent randomly selected specimens and subpopulations with low adherence to study medication, we comprehensively assessed PrEP sharing within this population. Re-randomization of placebo arm participants created a unique opportunity to assess drug sharing when all HIV-1 uninfected partners received active PrEP, conditions that could have incentivized drug sharing. Importantly, we did not find an increase in drug sharing post-unblinding.

\section{CONCLUSIONS}

Self-reported drug sharing in the Partners PrEP Study was extremely rare. Objective plasma tenofovir testing supports the self-reported data and indicates that sharing PrEP within HIV-1 serodiscordant couples was uncommon. These results suggest that it is unlikely for medication sharing to be a serious limitation for PrEP delivery to HIV-1 uninfected members of HIV-1 serodiscordant couples.

\section{ACKNOWLEDGMENTS}

The authors thank the couples who participated in this study and the teams at the study sites for work on data collection and management.

\section{Partners PrEP Study Team.}

University of Washington Coordinating Center and Central Laboratories: Connie Celum (principal investigator, protocol co-chair), J.M.B (medical director, protocol cochair), Deborah Donnell (protocol statistician), Robert W. Coombs, Lisa Frenkel, Craig W. Hendrix, Jairam Lingappa, M. Juliana McElrath.

Study sites and site principal investigators: Eldoret, Kenya (Moi University, Indiana University): Kenneth Fife, Edwin Were; Kabwohe, Uganda (Kabwohe Clinical Research Center): Elioda Tumwesigye; Jinja, Uganda (Makerere University, University of Washington): P.N., Elly Katabira; Kampala, Uganda (Makerere University): Elly Katabira,
Allan Ronald; Kisumu, Kenya (Kenya Medical Research Institute, University of California San Francisco): Elizabeth Bukusi, Craig Cohen; Mbale, Uganda (The AIDS Support Organization, CDC-Uganda): Jonathan Wangisi, James Campbell, Jordan Tappero; Nairobi, Kenya (University of Nairobi, University of Washington): James Kiarie, Carey Farquhar, Grace John-Stewart; Kenya Medical Research Institute, Nairobi, Kenya: Nelly Rwamba Mugo; Tororo, Uganda (CDC-Uganda, The AIDS Support Organization): James Campbell, Jordan Tappero, Jonathan Wangisi.

Data management was provided by DF/Net Research, Inc. (Seattle, USA) and site laboratory oversight was provided by Contract Laboratory Services (University of the Witwatersrand, Johannesburg, South Africa). Study medication donated by Gilead Sciences, Inc.

\section{REFERENCES}

1. World Health Organization (WHO). Guidance on Oral Pre-Exposure Prophylaxis (PrEP) for Serodiscordant Couples, Men and Transgender Women Who Have Sex with Men at High Risk of HIV: Recommendations for Use in the Context of Demonstration Projects. Geneva: World Health Organization; 2012.

2. McCormack S, Dunn DT, Desai M, et al. Pre-exposure prophylaxis to prevent the acquisition of HIV-1 infection (PROUD): effectiveness results from the pilot phase of a pragmatic open-label randomised trial. Lancet. 2016;387:53-60.

3. Marcus JL, Volk JE, Pinder J, et al. Successful implementation of HIV preexposure prophylaxis: lessons learned from three clinical settings. Curr HIV/AIDS Rep. 2016;13:116-124.

4. Liu AY, Cohen SE, Vittinghoff E, et al. Preexposure prophylaxis for HIV infection integrated with municipal- and community-based sexual Health Services. JAMA Intern Med. 2016;176:75-84.

5. Grant RM, Anderson PL, McMahan V, et al. Uptake of pre-exposure prophylaxis, sexual practices, and HIV incidence in men and transgender women who have sex with men: a cohort study. Lancet Infect Dis. 2014; $14: 820-829$

6. Baeten JM, Heffron R, Kidoguchi L, et al. Integrated delivery of antiretroviral treatment and pre-exposure prophylaxis to HIV-1serodiscordant couples: a prospective implementation study in Kenya and Uganda. PLoS Med. 2016;13:e1002099.

7. Grelotti DJ, Closson EF, Mimiaga MJ. Pretreatment antiretroviral exposure from recreational use. Lancet Infect Dis. 2013;13:10-12.

8. Surratt HL, Kurtz SP, Cicero TJ, et al. Antiretroviral medication diversion among HIV-positive substance abusers in South Florida. Am J Public Health. 2013;103:1026-1028.

9. Kurtz SP, Buttram ME, Surratt HL. Vulnerable infected populations and street markets for ARVs: potential implications for PrEP rollout in the USA. AIDS Care. 2014;26:411-415.

10. World Health Organization (WHO). Consolidated Guidelines on the Use of Antiretroviral Drugs for Treating and Preventing HIV Infection. Geneva: World Health Organization; 2013.

11. Katzenstein DA, Hammer SM, Hughes MD, et al. The relation of virologic and immunologic markers to clinical outcomes after nucleoside therapy in HIV-infected adults with 200 to $500 \mathrm{CD} 4$ cells per cubic millimeter. AIDS Clinical Trials Group Study 175 Virology Study Team. N Engl J Med. 1996;335:1091-1098.

12. Baeten JM, Donnell D, Mugo NR, et al. Single-agent tenofovir versus combination emtricitabine plus tenofovir for pre-exposure prophylaxis for HIV-1 acquisition: an update of data from a randomised, doubleblind, phase 3 trial. Lancet Infect Dis. 2014;14:1055-1064.

13. Baeten JM, Donnell D, Ndase P, et al. Antiretroviral prophylaxis for HIV prevention in heterosexual men and women. $N$ Engl J Med. 2012;367: 399-410.

14. Ndase P, Celum C, Campbell J, et al. Successful discontinuation of the placebo arm and provision of an effective HIV prevention product after a positive interim efficacy result: the partners PrEP study experience. $J$ Acquir Immune Defic Syndr. 2014;66:206-212. 
15. Haberer JE, Baeten JM, Campbell J, et al. Adherence to antiretroviral prophylaxis for HIV prevention: a substudy cohort within a clinical trial of serodiscordant couples in East Africa. PLoS Med. 2013;10:e1001511.

16. Bushman LR, Kiser JJ, Rower JE, et al. Determination of nucleoside analog mono-, di-, and tri-phosphates in cellular matrix by solid phase extraction and ultra-sensitive LC-MS/MS detection. J Pharm Biomed Anal. 2011:56:390-401.

17. Hendrix CW, Andrade A, Bumpus NN, et al. Dose frequency ranging pharmacokinetic study of tenofovir-emtricitabine after directly observed dosing in healthy volunteers to establish adherence benchmarks (HPTN 066). AIDS Res Hum Retroviruses. 2016;32:32-43.

18. Musinguzi N, Muwonge T, Thomas K, et al. Does adherence change when No one is Looking? Comparing announced and unannounced tenofovir levels in a PrEP trial. AIDS Behav. 2016;20:2639-2643.
19. Donnell D, Baeten JM, Bumpus NN, et al. HIV protective efficacy and correlates of tenofovir blood concentrations in a clinical trial of PrEP for HIV prevention. J Acquir Immune Defic Syndr. 2014;66: 340-348.

20. Corneli AL, McKenna K, Perry B, et al. The science of being a study participant: FEM-PrEP participants' explanations for overreporting adherence to the study pills and for the whereabouts of unused pills. J Acquir Immune Defic Syndr. 2015;68:578-584.

21. Kahle EM, Kashuba A, Baeten JM, et al. Unreported antiretroviral use by HIV-1-infected participants enrolling in a prospective research study. J Acquir Immune Defic Syndr. 2014;65:e90-e94.

22. Fogel JM, Wang L, Parsons TL, et al. Undisclosed antiretroviral drug use in a multinational clinical trial (HIV Prevention Trials Network 052). J Infect Dis. 2013;208:1624-1628. 